\title{
Attenuation Characteristics of Laser in Rain
}

\author{
Yuewei Wei ${ }^{\mathrm{a}}$, Jun $\mathrm{Xu}^{\mathrm{b}}$ and Bin $\mathrm{Xu}$ \\ Xian Research Institute of High Technology, Xi'an 710025, China

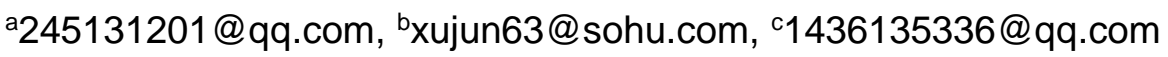

\begin{abstract}
In the observation of laser signal transmission in the rain, the attenuation ratio with the change of the size of the raindrops, the smaller the smaller the attenuation. In Mie scattering theory, with Weibull distribution model of raindrop size distribution and calculate the laser beam transmission in the rain attenuation characteristics in rainfall and changes with the distance, in Mie scattering theory, with Webb distribution model of raindrop size distribution, calculation of laser beam in the transmission of the rain attenuation characteristics in rainfall and changes with the distance. In the process of propagation, only the scattering attenuation of raindrops is considered, and the influence of diffraction and geometrical scattering is also considered. This explains the observed phenomena in the experiment, the attenuation of the laser signal in the light rain is larger than that in the heavy rain.
\end{abstract}

Keywords: Laser transmission; Rainfall attenuation; Mie scattering; Transmittance.

\section{Introduction}

With the laser radar, laser communication and laser guided weapons development and laser technology in national defense plays a more and more important role, the characteristics of laser transmission in the atmosphere is one of the most important problems in the military and civilian laser engineering system in atmospheric operation must be studied. Laser is not affected by the external electromagnetic field and the electrostatic induction, and there is no electronic interference in the radio system [1]. The direction of the laser beam is very strong, almost no side lobe, the beam divergence angle is very small, and so it has a good spatial orientation. Therefore, in order to overcome the influence of rainfall factors on laser detection system, it is needed to study the effect of different rainfall on the attenuation of laser signal.

\section{Characteristics and size distribution of raindrops}

The initial study of raindrop shape is from the angle of meteorology. Photographic studies show that the radius of less than $1 \mathrm{~mm}$ of the rain is basically spherical, for larger raindrops, the shape of the bottom of a groove of the flat ellipsoid, its rotation axis is similar to the vertical direction. The attenuation of atmospheric molecules is composed of absorption and scattering. Molecular absorption is a complex process, but it is closely related to the wavelength of light wave [2]. The raindrops spectrum is a necessary parameter to calculate the optical signal transmission in the rain, which is the introduction of the distribution of raindrops in the unit space with different sizes.

\subsection{Raindrops spectrum model}

Effects of raindrops on the light propagation depends mainly on the raindrop size distribution, scattering and absorption cross section. The raindrop size distribution is under a certain rainfall rate, different size raindrops in per unit volume of space distribution shape. Raindrop size and distribution in space is random, normally people use raindrop size distribution function $N(D)$ to describe the raindrop size distribution, the unit is $\mathrm{m}^{-3} \mathrm{~mm}^{-1} . D$ said raindrop diameter and unit is $\mathrm{mm}$. There is literature summarizes and points out that in many raindrop spectrum model, the M-P distribution model, Joss distribution model and Weibull distribution model is relatively accurate. It can be used to calculate the light attenuation in the rain, the Weibull distribution model of the general form can be applied to any region [3]. 


\subsection{Drop velocity}

Due to gravity, buoyancy and air resistance of the role of these three forces, the rain falling in the air to do accelerated movement. Along with the increase of the speed of the rain, the air resistance of the rain is getting bigger and bigger, at the same time, it will be getting smaller and smaller. When these three forces to achieve balance, the acceleration is equal to zero, then the rain will decline at a constant speed, this speed is the end of the drop in the rain.

By the drop velocity and other parameters, the rainfall can be calculated, and the distribution of the size distribution of raindrops can also be obtained [4]. On the basis of the experimental data and analysis of other people's data and analysis, the empirical formula of Hale and Querry is obtained:

$v(D)=9.65-10.3 e^{-0.6 D}$

In the formula, $D$ is the equivalent diameter of raindrops, whose unit is $\mathrm{mm}$.

\section{Law of laser attenuation}

Relative to the wavelength of light, raindrops are large particles, according to the following calculation, the rain attenuation should be independent of the wavelength. However, due to the raindrop induced light scattering with wavelength change; as gas molecules of water vapor content with the rainfall intensity will have varying degrees of change, and water vapor absorption with wavelength have drastic changes, so rain attenuation in different wavelength is different. Laser emitted by the laser range finder, after atmospheric transmission reach the target and laser signal reflected back to the rangefinder receiver by diffuse reflection to the, followed by signal acquisition system, the signal acquisition and storage, for later analysis.

\subsection{Mie scattering theory}

Mie theory is a rigorous theory to describe the light scattering of spherical particles. It is only assumed that the particle material is optically homogeneous and isotropic, and can be derived from the Maxwell equations and the boundary conditions [5]. So it is suitable to describe the light scattering of spherical particles of any size. Its formula is:

$$
Q_{\mathrm{e}}=\frac{2}{\alpha^{2}} \operatorname{Re} \sum_{m=1}^{\infty}(2 m+1)\left(a_{m}+b_{m}\right)
$$

In the formula, $\alpha$ represents the particle size parameter, $\alpha=2 \pi r / \lambda . a_{\mathrm{m}}, b_{\mathrm{m}}$ is the Mie scattering coefficient, and its formula is:

$$
\begin{aligned}
& a_{m}=\frac{\Psi_{m}^{\prime}(n \alpha) \Psi_{m}(\alpha)-n \Psi_{m}(n \alpha) \Psi_{m}^{\prime}(\alpha)}{\Psi_{m}^{\prime}(n \alpha) \mathrm{Z}_{m}(\alpha)-n \Psi_{m}(n \alpha) \mathrm{Z}_{m}^{\prime}(\alpha)} \\
& b_{m}=\frac{n \Psi_{m}^{\prime}(n \alpha) \Psi_{m}(\alpha)-\Psi_{m}(n \alpha) \Psi_{m}^{\prime}(\alpha)}{n \Psi_{m}^{\prime}(n \alpha) \mathrm{Z}_{m}(\alpha)-\Psi_{m}(n \alpha) \mathrm{Z}_{m}^{\prime}(\alpha)} \\
& \Psi_{m}(\rho)=\rho j_{m}(\rho)=\sqrt{\frac{\pi \rho}{2}} J_{m+1 / 2}(\rho) \\
& \mathrm{Z}_{m}(\rho)=\rho h_{m}^{(2)}(\rho)=\sqrt{\frac{\pi \rho}{2}} H_{m+1 / 2}(\rho)
\end{aligned}
$$

In the formula, $n$ is the refractive index, and the frequency of the laser light. $J_{\mathrm{m}+1 / 2}(\rho)$ is the Bessel function, $H_{\mathrm{m}+1 / 2}(\rho)$ is Hankel function.

\subsection{Beer-Lambert Law}

Beer-Lambert Law is the basic law of light absorption, applicable to all electromagnetic radiation and all the ceiling light material, including gases, solids, liquids, and molecules, atoms and ions[6]. Beer-Lambert Law is law and the photoelectric colorimetric quantitative based colorimetric spectrophotometric method, analysis. The amount of light absorbed is proportional to the number of molecules generated in the optical path of the light absorption: 


$$
T=\frac{P_{t}}{P_{0}}=e^{-\beta L}
$$

Where $T$ is the laser transmittance, said transmission light intensity and the incident light intensity ratio, reaction of the laser energy attenuation degree; $P_{\mathrm{t}}$ and $P_{0}$ were transmitted light intensity and the incident light intensity; $L$ for laser transmission distances in the rain; $\beta$ is the extinction coefficient.

\section{Simulation and verification of laser transmission in the rain}

It is also subject to the influence of the scattering of the optical geometry, which can establish the model of the attenuation of the green laser energy transfer from the single dispersed system [7]. Thus currently used some atmospheric transmission software to calculate the wave radiative transfer properties of software integration of a variety of atmospheric model and calculation method can be more comprehensively calculated spectral transmittance, the current relatively mature have LOWTRAN and MODTRAN.

\subsection{Simulation of laser transmission in the rain}

Figure 1 shows the three types of precipitating clouds mean spectrum of the fitting results, on average spectral fitting results show that the two distributions underestimate the number of droplets, the fitting effect of medium size raindrops are very good, in the middle section of the distribution function of the difference influence the fitting effect is very small, even if is the precipitation cumulonimbus cloud and stratiform mixed cloud. In comparison, the Joss distribution of the average spectral representation is better, especially in a droplet.

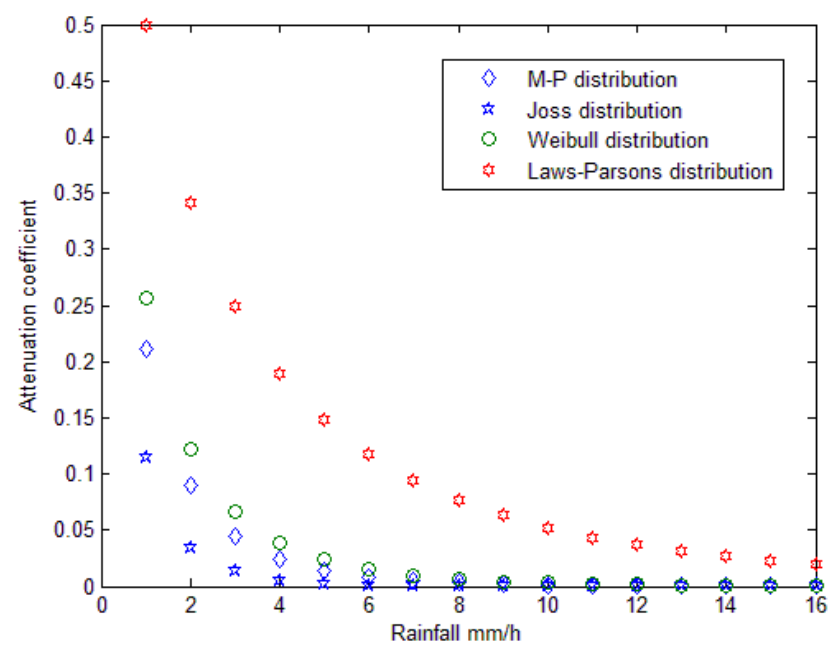

Fig.1 Attenuation coefficient of laser in rain

However, the actual drop spectrum and the average spectrum deviation in the precipitation process is larger, and the average spectrum cannot completely represent the actual case spectrum. Figure 1 is the error sequence of the output from the low to high order according to the measured data. From the fitting results of number concentration, Weibull model is more close to the measured value, and fitting higher stability. M-P fitted value in number concentration is low it intends to values higher than the measured value, but in the number concentration is high, the fitted values should be lower than the measured value, the concentration coefficient depending on the droplets.

\subsection{Verification of simulation results}

As can be seen from Figure 2, the experimental results are consistent with the general trend of software calculation and empirical formula calculation results, which verify the rationality of the experimental data processing. Figure shows that with enhanced visibility, atmospheric for 1.06um laser attenuation coefficient is more and more small indicates laser transmittance becomes higher and higher. Therefore, the use of laser equipment in good visibility weather conditions is very favorable. Comparative analysis of four curves can be found, in visibility for $5 \sim 10 \mathrm{~km}$ of the conditions, the experimental data and the software calculation data and empirical formula calculation data are 
agreement with better, the relative error between does not exceed $10 \%$ of the, especially in visibility $5 \mathrm{~km}$ conditions for minimum error less than $6 \%$, indicating that the atmospheric transmission software with the empirical formula in visibility is less than $5 \mathrm{~km}$ good weather of 1.0um laser is applicable and has high accuracy. With decreasing visibility, especially in visibility below $3 \mathrm{~km}$, has been able to see the curve has obvious difference, especially the empirical formula for calculating the data, when visibility $2 \mathrm{~km}$ and $1 \mathrm{~km}$ the deviation has reached more than $50 \%$, indicating that for $\mathrm{M}$ laser, empirical formula in poor visibility conditions can not accurately reflect the atmospheric attenuation coefficient changes, and software calculation results and the experimental results with relatively good.

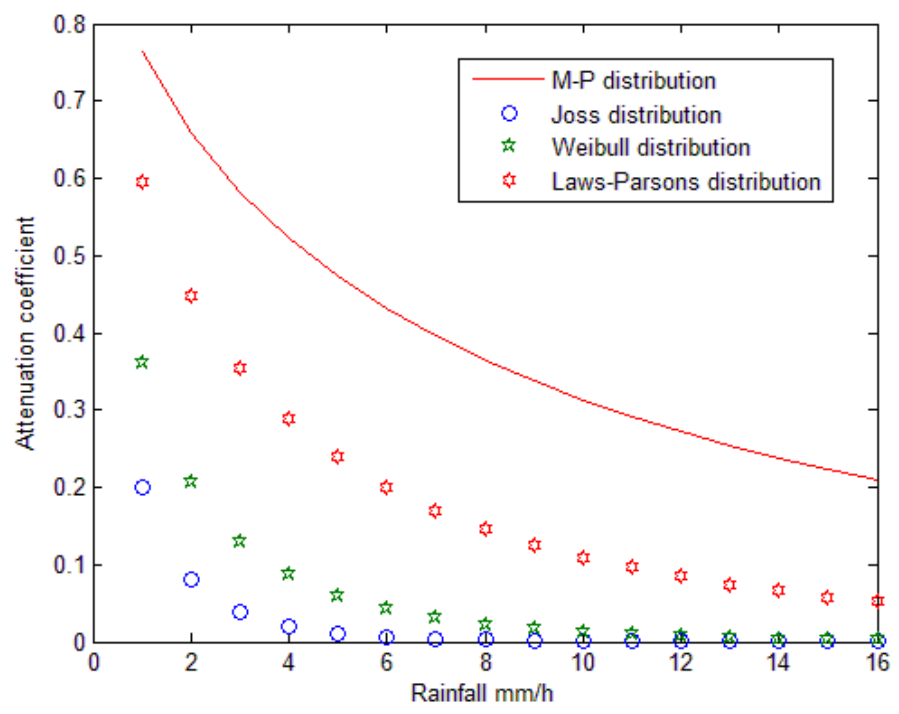

Fig.2 Verification of attenuation coefficient of laser in rain

\section{Summary}

Application of Mie theory, analyzes the single ball rain particle on the laser scattering, scattering diagrams show that for a large scale particle like raindrops, scattering light is mainly concentrated in the former and the latter to, and the scattering intensity of backward and to a point of relatively narrow. From the previous analysis, we can see that the scattering of raindrops has a very important effect on the attenuation of laser in the rain. Study and analysis show that the absorption rate of the raindrops on the wavelength of 1.06 um laser is $1.32 \times 10-9$, which can be ignored. In raindrops scattering process, due to diffraction attenuation with raindrop size and does not consider the influence of refractive index and when the scattering angle is increased, the Fraunhofer diffraction exist large errors, while considering the influence of geometric optics, establishing the attenuation cross section model and Mie scattering theory similar conclusions and simplify the complicated calculation process. According to the experimental data, it is shown that, after the rain scattering, light energy is mainly concentrated in the former to within a certain angle, the large angle scattering light is smaller, and it increases with increase of diameter of raindrop, the experimental results and the theoretical calculation are basically the same, the maximum error is less than $2 \%$. It is proved that the correctness of the theoretical model. This provides a theoretical basis for the application of laser in the rain environment.

\section{References}

[1] Guo Jing. Study on the transmission characteristics of pulse laser for near-field fixed-distance in the rain. Nanjing: Nanjing University of Science and Technology, 2012

[2] Cheng Jinbo. Study on the analyses and calculate of rain attenuation of Ka-band satellite communication system. Xi'an: Xidian University, 2014 
[3] Wang Duo, Han Yanli, Sun Tengfei. Analysis of influence from rain and fog on star target detection during daytime. Electro-Optic Technology Application, 2013, 28(6): 74-76

[4] Sun Xuejin, Sun Haiyang, Jiang Zhidong. Numerical simulation of terminal velocity of raindrops falling under different atmospheric conditions. Computer Simulation, 2011, 28(12): 402-406

[5] Li Weimin, Ao Faliang, Yu Shengyun. Study of forward scattering effections on laser transformation in raindrop. Photon Technolog, 2006(4): 237-240

[6] Gao Guoqiang. Study of laser propagation and attenuation through the rain and fog environment. Xi' an: Xi'an University of Science and Technology, 2014

[7] Wang Linlin, Duan Jin, Fu Jun, et al. Analysis on the impact of rainfall on laser transmission distance. Applied Laser. 2015, 35(1): 99-103. 